\title{
In-house preparation of hydrogels for batch affinity purification of glutathione $S$-transferase tagged recombinant proteins
}

\author{
Jason S Buhrman ${ }^{1}$, Jamie E Rayahin ${ }^{1}$, Melanie Köllmer ${ }^{1}$ and Richard A Gemeinhart ${ }^{1,2,3^{*}}$
}

\begin{abstract}
Background: Many branches of biomedical research find use for pure recombinant proteins for direct application or to study other molecules and pathways. Glutathione affinity purification is commonly used to isolate and purify glutathione S-transferase (GST)-tagged fusion proteins from total cellular proteins in lysates. Although GST affinity materials are commercially available as glutathione immobilized on beaded agarose resins, few simple options for in-house production of those systems exist. Herein, we describe a novel method for the purification of GST-tagged recombinant proteins.
\end{abstract}

Results: Glutathione was conjugated to low molecular weight poly(ethylene glycol) diacrylate (PEGDA) via thiol-ene "click" chemistry. With our in-house prepared PEGDA:glutathione (PEGDA:GSH) homogenates, we were able to purify a glutathione S-transferase (GST) green fluorescent protein (GFP) fusion protein (GST-GFP) from the soluble fraction of E. coli lysate. Further, microspheres were formed from the PEGDA:GSH hydrogels and improved protein binding to a level comparable to purchased GSH-agarose beads.

Conclusions: GSH containing polymers might find use as in-house methods of protein purification. They exhibited similar ability to purify GST tagged proteins as purchased GSH agarose beads.

Keywords: Glutathione, PEGDA, Glutathione S-transferase, Batch purification, Recombinant protein

\section{Background}

Recombinant DNA and protein technologies have taken a leading role in many forms of research over the past 40 years [1]. Recombinant proteins have found their way to the core of most biomedical research. Classical methods of protein purification can be divided into methods that utilize chemical properties of the protein including solubility [2], physical characteristics, e.g. isoelectric points and size [3], and those that use selective endogenous or engineered [4] protein affinity. Of this latter category, fusion proteins have emerged as a prominent method for purification. Fusion proteins are created by appending a full length or truncated protein to a terminal region of the protein of interest. For affinity fusion

\footnotetext{
* Correspondence: rag@uic.edu

'Department of Biopharmaceutical Sciences, University of Illinois, Chicago, IL 60612-7231, USA

2Department of Bioengineering, University of Illinois, Chicago, IL 60607-7052, USA

Full list of author information is available at the end of the article
}

proteins, the appended protein will bind to a third, typically small, molecule that is immobilized on the surface of a polymer resin. The fusion protein will selectively bind to the surface while other proteins are washed away [5]. In column chromatography methods, protein lysates are eluted through columns that are packed with polymer particles. Alternatively, the affinity matrix can be mixed directly with the protein lysates, agitated for some amount of time, centrifuged and collected in batch selection methods. Fusion proteins are genetically tagged with a protein of known affinity. The glutathione $\mathrm{S}$-transferase (GST)-protein is frequently used to tag a protein of interest because of its affinity for the reduced form of the tripeptide, glutathione [6]. During the elution process, excess glutathione is added to remove the tagged protein from the affinity matrix.

Traditionally, glutathione is covalently linked to the surface of activated agarose beads $\left(\right.$ Sepharose $\left.{ }^{\circledR}\right)$. A thiol bond is formed between the glutathione and an alipathic spacer that is linked to a hydroxyl group on the surface 
of the agarose bead [7]. Since the inception of using glutathione as a capture agent for GST fusion proteins, almost all scientific supply companies carry a variation of glutathione conjugated polymeric beads, and at least one patent has been awarded [8] for its utility in proteins. Agarose has been widely used for glutathione conjugation. The chemistry of this linkage has multiple reaction and purification steps that restrict in-house production of these beads to chemistry laboratories $[4,7]$.

Thiol-ene "click" chemistry has been shown to reproducibly form covalent thioether bonds between thiol and alkene-containing molecules. Glutathione (GSH) has been covalently linked to alkene groups in poly ( $\mathrm{N}$-isopropyl acrylamide) (PNIPAm) polymers [9]. While glutathione linkage to PNIPAm was confirmed, a detailed validation of the interaction with GST tagged proteins was not presented.

Using a modified form of thiol-ene chemistry and readily available materials, we demonstrate a simple, one-step method for creating gel homogenates and beads with affinity toward GST tagged proteins. Specifically, our method employs thiol-ene addition of glutathione to low molecular weight poly(ethylene glycol) diacrylate (PEGDA). Under standard conditions, the resulting polymer forms hydrogels with typical radical initiators. These gels were readily homogenized, washed, and used to purify soluble GST proteins. In our proof of concept study, GST-fused, red shifted green fluorescent protein (rsGFP) was purified with PEGDA:GSH homogenates. We also demonstrate a novel method for creating glutathione-laden PEGDA microspheres using reversephase emulsion polymerization. Like the homogenates, PEGDA:GSH microspheres exhibited affinity to GST-GFP and can be used to purify the protein. Either of these methods can be implemented in almost any laboratory using readily available, inexpensive reagents.

\section{Results and discussion}

Challenging economic times combined with the influx of new scientists in biomedical research makes funding opportunities less frequent and resources increasingly strained. In response to these environmental pressures, scientists must adapt by using any available resources as tools for discovery. Newer, cheaper methods will replace traditionally more expensive techniques. Although many kits are available, most of these kits have substantial mark-up that is not always combined with quality.

To create a low-cost approach using widely available laboratory chemicals for purifying GST tagged proteins, we have utilized thiol-ene addition of reduced glutathione to low molecular weight PEGDA. We chose to look predominately at homogenization of gels as a batch purification method because most laboratories have access to plastic eppendorf homogenizers or similar. This simple method was shown to be reproducible and effective. We further develop a more complex method that has more uniformity and compares well with commercially available GST affinity beads.

\section{Creation of vectors \& protein expression}

To create a model protein for purification, we chose to use the GFP for its solubility and ease of visualization [10]. The vector, pET 15b (Figure 1A-1), was constructed by inserting a flexible spacer sequence, a hexahistadine tag and thrombin cleavable sequence (Figure 1B, top). The sequence was isolated by PCR (Figure 1A-2) and cloned into pGEX 6p-1 adjacent to the GST sequence (Figure 1A-3). GFP was isolated from gWIZ-GFP by PCR that added a separate spacer region and a second hexa-histadine tag (Figure 1A-4). GFP was inserted into the pGEX vector for expression (Figure 1A-5 \& 6). The second hexa-histadine tag of the protein was not utilized in these experiments, but is relevant and must be noted because Ni-NTA was used to purify the GST-GFP for several of the experiments being discussed. The effect of dual hexahistadine tags had on purification of the GST-GFP protein was not analyzed. It is clear that these dual tags did not negatively impact the protein activity (fluorescence) or binding capability to GSH. Resulting colonies were screened for gross fluorescence of the colonies (Figure 1C, top) and sequenced. GST-GFP was readily observed (Figure 1C, lower panel) and purified with a yield of approximately $300 \mathrm{mg} / \mathrm{L}$ of culture (data not shown). The nickel purified protein fraction was run on a SDS-PAGE gel to confirm size and thrombin cleavability (Figure 1D). The functionality of GST-GFP protein was confirmed by its increased affinity to nickel (data not shown) and glutathione beads, its green color and fluorescence, and its cleavability by thrombin. With our model protein produced and validated, we proceeded to validate the purification process.

\section{Affinity of GST-GFP to hydrogel homogenates}

Hydrogels were made as described with varying PEGDA to GSH ratios, and the gels were homogenized, washed, and incubated with $60 \mu \mathrm{g}$ of GST-GFP for $2 \mathrm{~h}$. When purified protein was incubated with the gels (Figure 2), a significant decrease in GST-GFP was found in the solution after two hours compared to gel-free controls in the $1: 1 \quad(\mathrm{p}=0.008), \quad 5: 1 \quad(\mathrm{p}=0.005), \quad 20: 1 \quad(\mathrm{p}=0.02), \quad 40: 1$ $(\mathrm{p}=0.02)$, and 80:1 $(\mathrm{p}=0.02)$ PEGDA to glutathione ratio gels (Figure 2A). There was clear increase in bound purified GST-GFP protein $(\mathrm{p}=0.00002$; Figure $2 \mathrm{~B})$ when GSH was incorporated in the gels at the highest extent. The 1:1 and 5:1 ratio gels were statistically different from all other groups while the lower incorporation ratios did not associate with significantly differing amounts of 


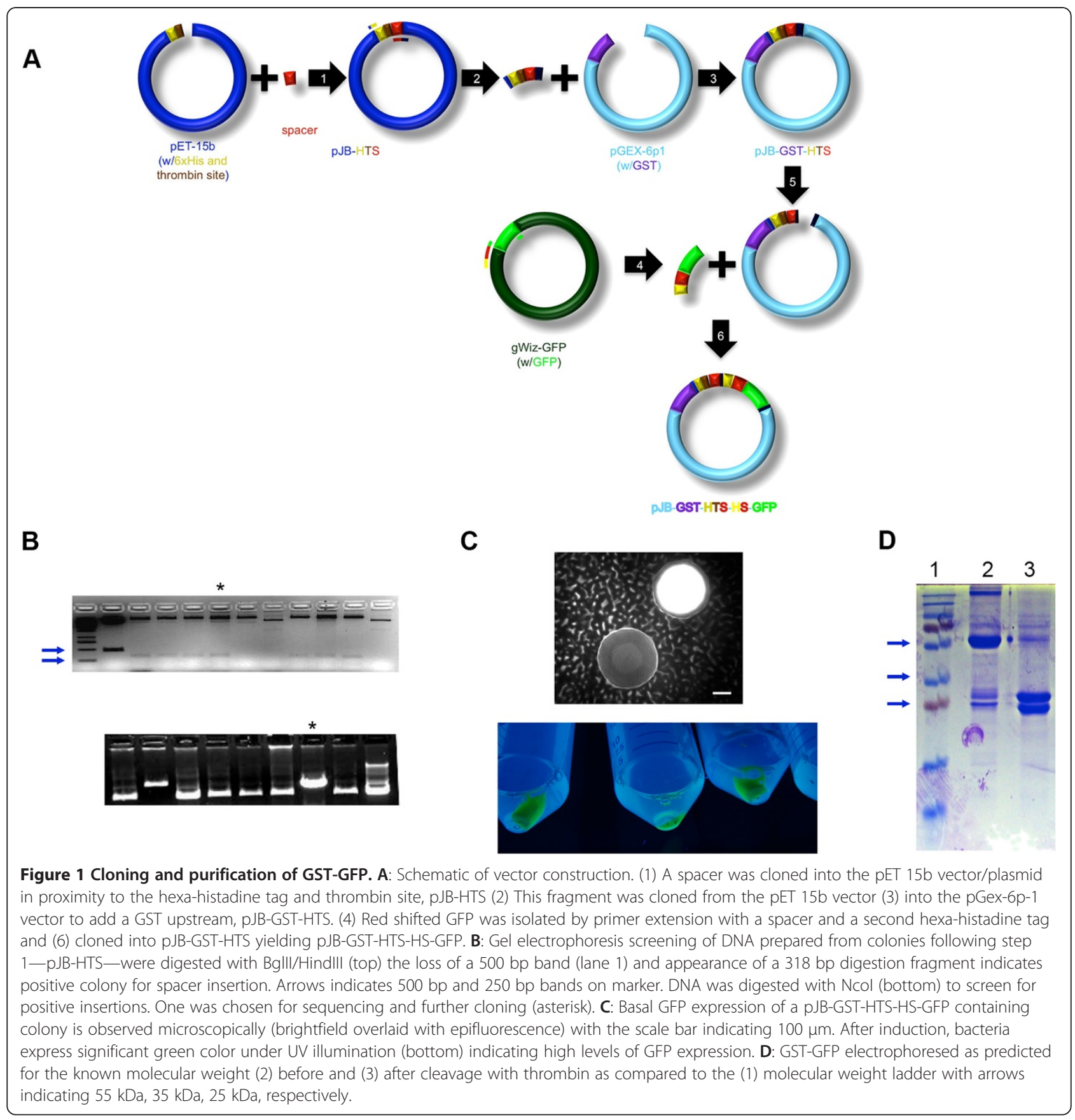

GST-GFP. These experiments demonstrate significant protein association with gel homogenates harboring GSH compared to controls. As the PEGDA:GSH ratio decreased, more GST-GFP was able to associate with the homogenized gels.

After homogenization and washing, PEGDA:GSH homogenates were used to specifically purify GST tagged GFP. Further, we demonstrated that decreasing the PEGDA:GSH ratio from 80:1 to 1:1 resulted in increased GST affinity to gel homogenates. The PEGDA:GSH ratio was not further optimized because GSH inhibits polymerization reactions and gel formation at higher concentration. GSH is a known radical scavenger that decreases the number of free radicals available to sustain the polymerization cycle [11]. GSH also incorporates into acrylate groups and stops chain growth by eliminating alkene groups necessary for polymer propagation [9]. At some point the PEGDA:GSH ratio will become low enough that no usable gel will form. In this report, we focused on gels that had qualitatively acceptable and manageable physical properties, i.e. handlability for homogenization. 


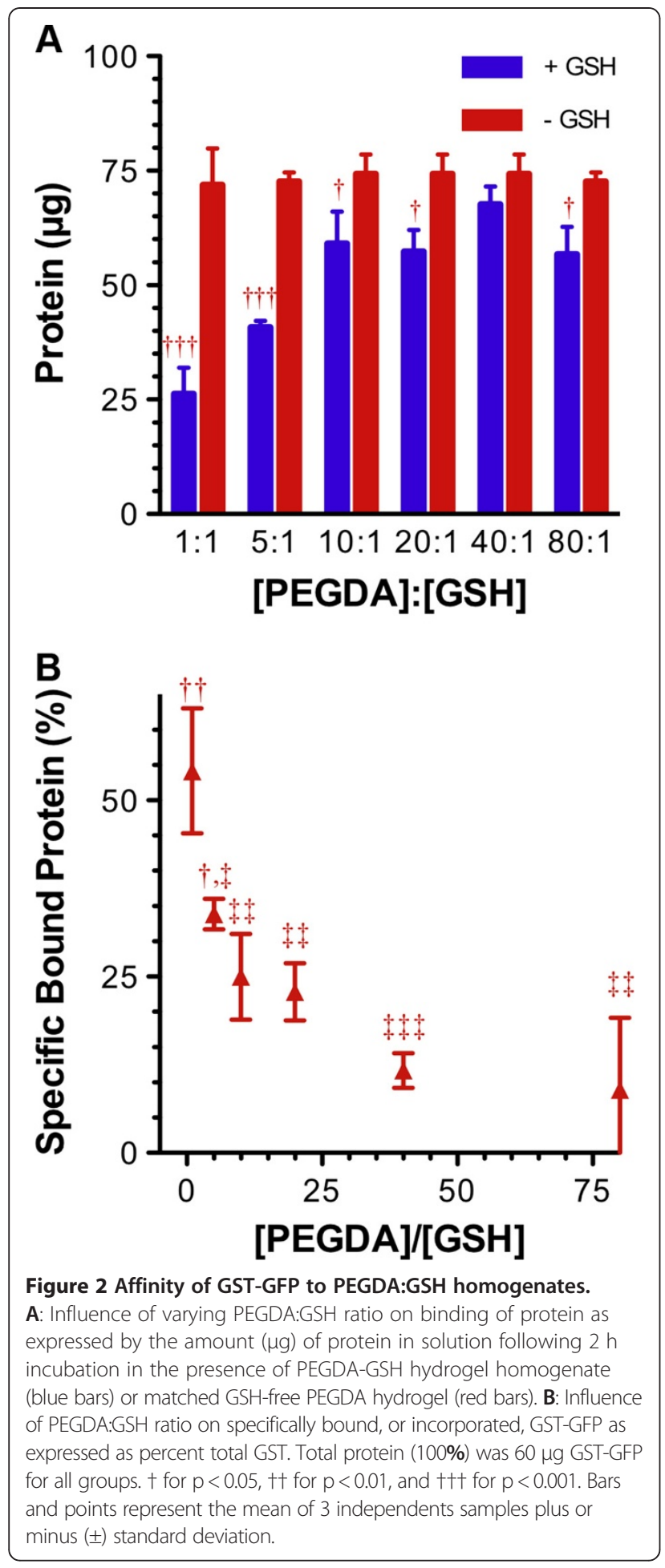

\section{Specificity of GST-GFP to GSH laden hydrogel} homogenates

To confirm the specificity of the interaction, nickelpurified GST-GFP was incubated in the presences of homogenates made with equimolar GSH (Figure 3A, top) or cysteine (Figure 3A, bottom). There was no

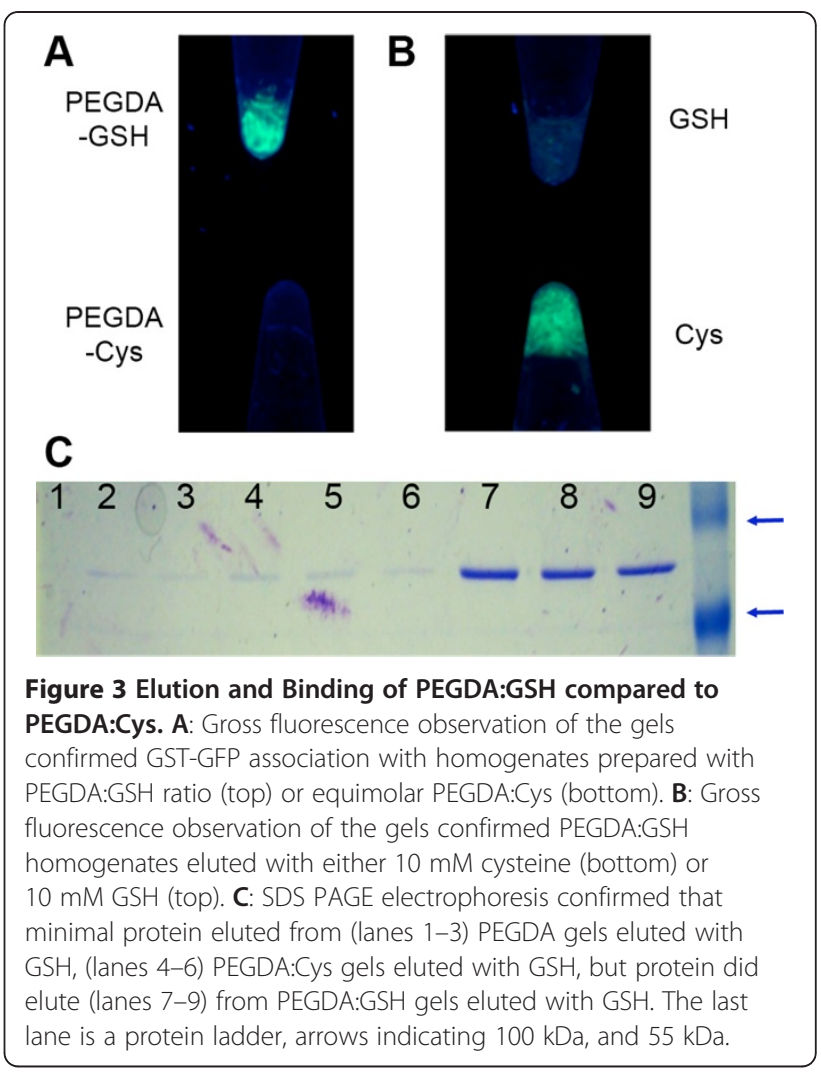

appreciable GFP observed in the cysteine-containing gels while the GSH-containing gels were visibly fluorescent. For homogenates made with glutathione, $10 \mathrm{mM}$ GSH (Figure 3B, top) was able to elute the GFP completely while $10 \mathrm{mM}$ cysteine (Figure $3 \mathrm{~B}$, bottom) was unable to elute the protein. Elution of the GST-GFP was found to be specific to GSH. No protein was eluted with GSH (Figure 3C, lanes 1-3) from hydrogels lacking GSH incorporation or eluted by cysteine (Cys; Figure 3C, lanes 4-6) from gels including glutathione. Proteins of appropriate molecular weight (Figure 3C, lanes 7-9) were eluted with GSH from GSH-containing gels.

\section{Purifying GST-GFP from $E$ Coli protein extract}

These experiments indicate that specific interactions take place between the gels and GST fusion proteins. This is not necessarily indicative of the ability of the materials to purify proteins from total bacterial lysates. Using the homogenized 5:1 PEGDA:GSH gels, crude lysates including GST-GFP from the induced, soluble protein fraction was batch selected over $2 \mathrm{~h}$ and eluted with $10 \mathrm{mM}$ GSH. From soluble lysate, it was difficult to elucidate the GST-GFP protein (Figure 4A, lane 1). Following nickel purification (Figure 4A, lanes 2 and 3), the enriched fraction showed a high degree of purity and appropriate size as a monomer in absence of glutathione (lane 2) or as a dimer in the presence of glutathione 


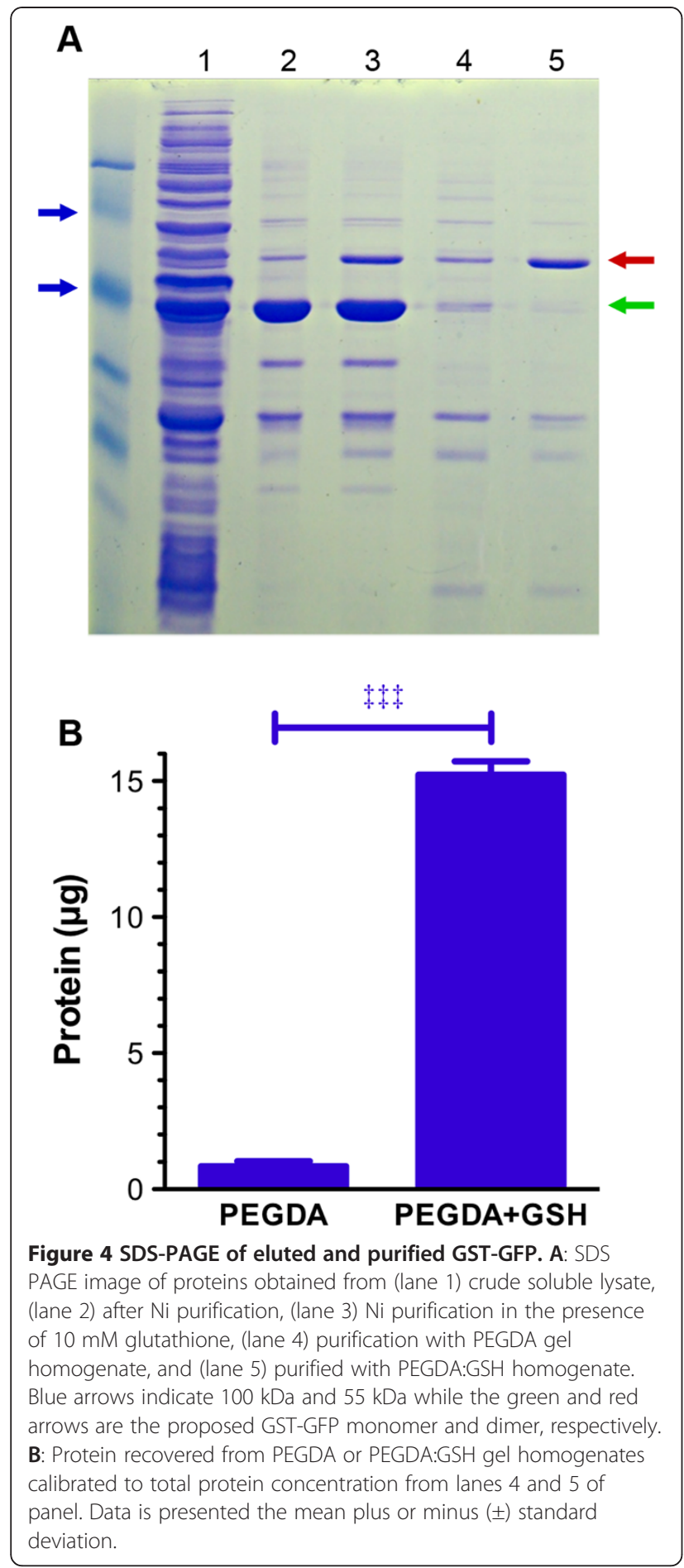

(lane 3). Without GSH incorporation, little protein and no purification was achieved with PEGDA gels (Figure 4A, lane 4). A significant single predominant band was obtained when purification took place in the presence of PEGDA:GSH gels (Figure 4A, lane 5). The size of nickel purified GST-GFP eluted with imidazole (Figure 4A, green arrow) is half the size of GST-GFP eluted with glutathione (Figure 4A, red arrow). A protein of this mass is present in all samples and being twice of the size of the GST-containing monomer suggests dimerization. The absence of the GST monomer with excess GSH further suggests that the dimer would be the predominant protein present. It is known that the GST acts as a homodimer with its substrate between the two monomers [12]. From $1 \mathrm{~mL}$ of the initial soluble GST-GSH lysate associated with the PEGDA:GSH homogenates, $0.8 \mu \mathrm{g}$ were eluted from the PEGDA homogenates, and $15 \mu \mathrm{g}$ were eluted from the PEGDA: GSH homogenates. This indicates an approximate 20 fold increased affinity of the GST-GFP to 5:1 PEGDA: GSH compared to PEGDA.

The homogenates were examined with fluorescence microscopy (Figure 5A) and it was noted that the areas of fluorescence differed in intensity inversely correlating with the thickness of the homogenized piece. Further, there was expected variation between the size of the

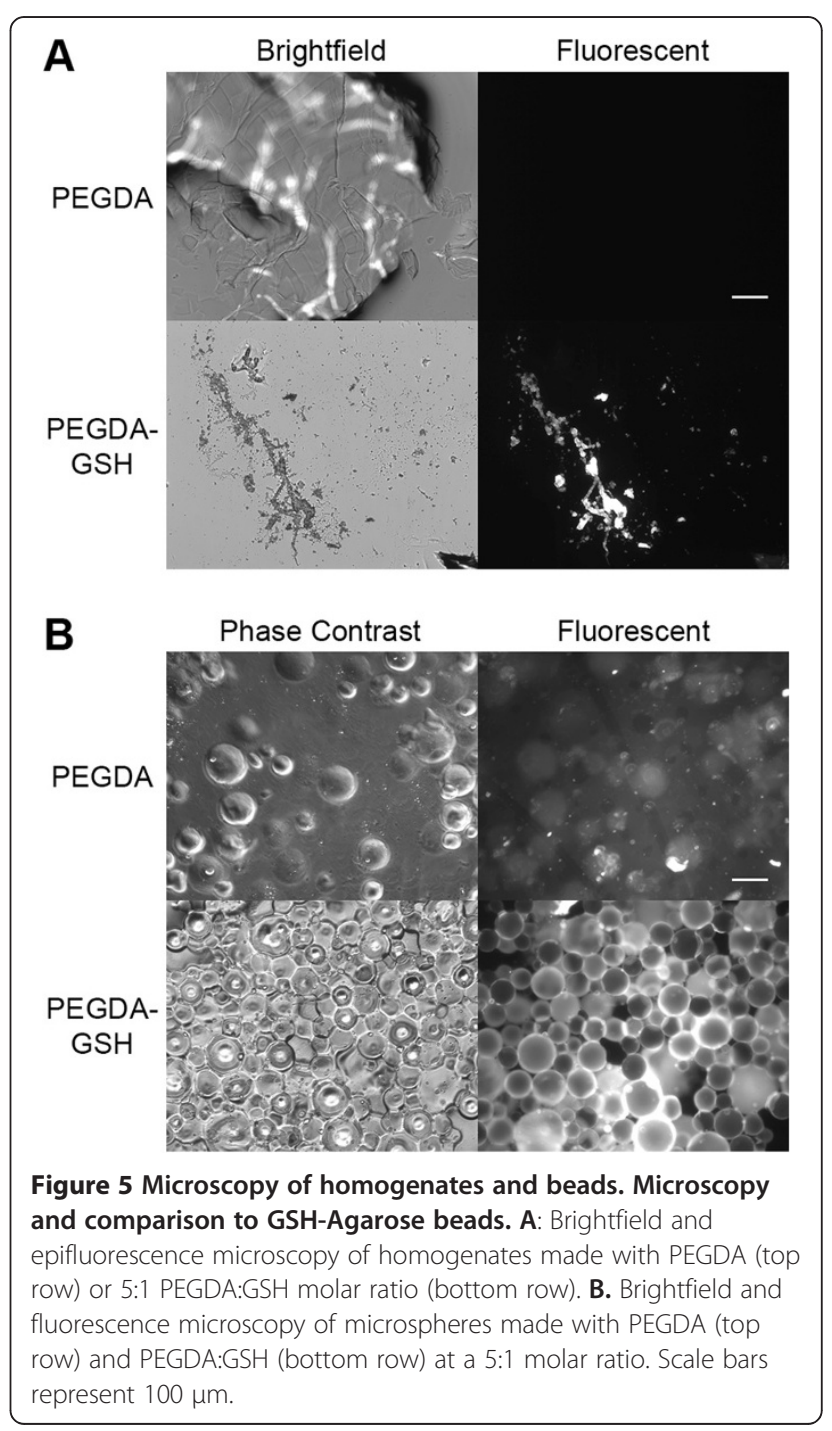


homogenized pieces that were between $10 \mu \mathrm{m}$ and $500 \mu \mathrm{m}$ (data not shown). Although we felt the homogenization method may be acceptable for labs with the facilities to make the particles and that these experiments allowed us to optimize the parameters necessary for protein purification, we felt that further improvements were possible. In order to improve the yield of GSH:PEGDA purified protein and consistency, we sought more homogeneously distributed, smaller particles as we expected these to allow for more efficient association of GST-GFP to the hydrogels.

\section{Creation of PEGDA:GSH microspheres and establishing affinity to GST-GFP}

We prepared microspheres using a reverse phase emulsion technique [13] to obtain more control over size and shape of the particles. The spheres were more homogenously distributed between $10 \mu \mathrm{m}$ and $200 \mu \mathrm{m}$ (Figure 5). In addition, spheres made with 5:1 PEGDA:GSH showed significant and uniform association with the GST-GFP (Figure 5B). Microspheres made from PEGDA:GSH had significantly greater affinity than PEGDA microspheres. In addition, there was no significant decrease in affinity to GST-GFP than purchased spheres made from GSHagarose (Figure 6; $\mathrm{P}=0.157$, one tailed student's t-test). We hypothesized that homogenous sized, spherical microspheres would increase GST-GFP association with the hydrogels by increasing the surface area of the polymer available for protein association. Microspheres were produced with PEGDA:GSH ratios as low as 5:1, but yields decreased at lower ratios (data not shown).

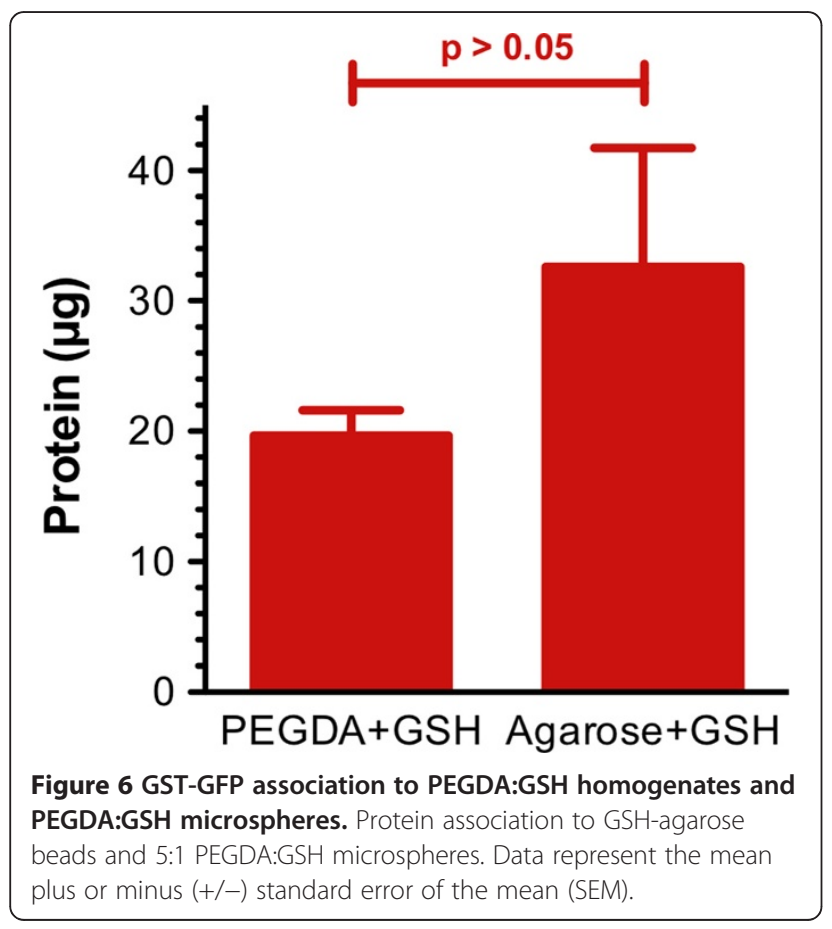

Spheres at a 5:1 PEGDA:GSH molar ratio displayed significant interaction with GST-GFP over control gels without GSH. These easy to fabricate and inexpensive particles have great potential for protein purification. This is particularly true since the microspheres interact similarly to purchased GSH-Sepharose ${ }^{\circledR}$ beads. Homogenates were able to remove $35 \%$ of the GST-GFP from the protein solution (Figure $2 \mathrm{~B}$ ) while microspheres bind a similar amount of GST-GFP (Figure 6) at 10 fold less mass. Microspheres demonstrate an increased binding capability compared to the homogenates. This increased binding may well be attributed to an increased surface area of the microspheres compared to homogenates, but further experiments would be needed to test this hypothesis.

Association of protein through GST-GSH interactions on the surface PEGDA microspheres introduces the exciting possibility of using these spheres for delivery of protein therapies. Building upon our previous work using proteolytically activated hydrogels [14-19], we envision using fusion proteins to release therapeutic proteins in response to disease-specific proteases. Therapeutic proteins would be released in the interstitial space [14] or intracellularly [20] by specific protease sites engineered between the GST and the therapeutic protein. Alternatively, high intracellular concentrations of reduced glutathione [21] could be used elute the GST-protein from the glutathione-containing material. Several proteases, including matrix metalloproteainse 2 (MMP-2), have been shown to be upregulated in many cancers $[22,23]$. Specific, short amino acid sequences have been discovered that are cleavable by MMP-2 [24-26], and could be incorporated between a tag GST and a therapeutic protein. In this way, the GST-GSH interaction could act as an anchor, holding the therapeutic protein on a hydrogel until MMP-2 cleaves the therapeutic protein from the GST anchor. Such an approach would be comparable to natural protein release from the extracellular matrix by MMPs. This biomimetic approach would advance the ability to bind protein therapeutics to hydrogels for disease-specific release. Further studies are underway to assess the potential for such a system in drug delivery.

\section{Conclusions}

We have shown a simple and effective method for conjugating glutathione to PEGDA. These materials specifically purify GST-tagged GFP. The purity of the GFP is similar to that purified by commercial $\mathrm{Ni}$-affinity agarose, and the purity and yield are compatible to purchased GST-Agarose beads. In challenging economic times we believe this simple and inexpensive method will be useful as an in-house alternative to purchasing 
more expensive commercial products in addition to other uses.

\section{Methods \\ Vectors}

All cloning was done in DH5alpha E. Coli cells and all vectors confer ampicillin resistance. The vector for protein production, pJB-GST-HTS-HS-GFP, was created by first cloning a 7 amino acid spacer fragment containing an XhoI site into pET-15b (Novagen) using sticky ligation and BamHI/XhoI sites. Colonies were mini-prepped by conventional SDS-precipitation and screened by BglII/HindIII (New England Biolabs; NEB) digestion (Figure 1B, lower panel), and confirmed by subsequent sequencing (ACGT). Primers were designed to fit in resulting vector, pJB-HTS upstream of the hexa-histadine tag (5/phos/CCATGGGCAGCAGC CATCATCAT), and downstream (AGCTGGAATTCC TAGTTATTGCTCAGCGGTGGC) (Integrated DNA technologies) of the spacer yielding a fragment containing a phosphorylated 5' end, an initiation codon, a hexa-histadine tag, a thrombin cleavable sequence, a spacer, a termination codon, and an EcoRI site. This fragment was digested with EcoRI (NEB) and ligated into pGex-6p1 (GE Healthcare) prepared by digestion with BamHI and blunting with Mung Bean Nuclease (NEB) to generate pJB-GST-HTS. Correct insertion yields a novel NcoI site generated by ligation of the blunted ends, and so NcoI (NEB) linearization of the supercoiled vector was used to determine correct insertion (Figure 1b, lower panel). These clones were confirmed by DNA sequencing. Primers were designed to clone rsGFP from gWIZ-GFP (Aldevron) and extending a BamHI site, a hexa-histadine tag, another spacer region, the GFP, and an EcoRI site. pJB-GST-HTS and the PCR fragment were prepared by BamHI and EcoRI digestion (Fw: AAAGGATCCATCATCATCATCATCA TGG TCCGC TGGGCGT TCG TG G TATGGCTAGCAA AGGAGAAGAACTC, Rev: AAAGAAT TCTCAGTT GTACAGTTCATCCATGCCATG). Colonies were screened under UV microscopy for basal expression of GFP (Figure 1c). DNA was sequenced from positive colonies containing the final product, pJB-GST-HTS-HS-GFP.

\section{Protein expression/Purification}

The vector, pJB-GST-HTS-HS-GFP was transformed into BL21 expression cells for expression and purification of GST-GFP protein. Protein was induced with $1 \mathrm{mM}$ IPTG after cells reached an absorbance of 0.5 at $595 \mathrm{~nm}$. They were removed to $25^{\circ} \mathrm{C}$ and were shaken overnight. Cells were spun at $4^{\circ} \mathrm{C}$ for $20 \mathrm{~min}$ to pellet, and resuspended in lysis buffer $(50 \mathrm{mM} \mathrm{NaH2PO}$, $300 \mathrm{mM} \mathrm{NaCl}, 10 \mathrm{mM}$ imidazole, $\mathrm{pH}=8$ ). The suspension was freeze-thawed $3 \mathrm{x}$ at $-80^{\circ} \mathrm{C}$, sonicated $3 \mathrm{x} 15 \mathrm{~s}$ then spun at $12 \mathrm{k} \mathrm{RPM}$ for 30 min to pellet the insoluble material. The supernatants were removed and selected through a gravity flow nickel column containing $1 \mathrm{~mL}$ of NTA Ni Agarose (Qiagen). After repeated washing the bound fraction was eluted with $250 \mathrm{mM}$ imidazole (Fischer) in lysis buffer containing 10\% glycerol (Acros).

\section{SDS-PAGE/Agarose gel electrophoresis}

All gels in this manuscript are 15\% w/v SDS PAGE gels made in-house with 37.5:1 acrylamide:bis (chemicals from sigma). Samples were prepared with Laemmeli sample buffer, and loaded into BioRad mini-protean II electrophoresis system at $150 \mathrm{v}$ until dye ran to end. Agarose gels were all $1.5 \% \mathrm{w} / \mathrm{v}$ agarose stained with ethidium bromide (Fischer) and imaged on BioRad GelDoc imager.

\section{Bradford assays}

Bradford assay was either purchased from Pierce or made in-house as described by Bradford 1972 [27]. Absorbances were routinely read $15 \mathrm{~min}$ after sample addition to G250. Homogenates were $50 \mathrm{mg}$ to $100 \mathrm{mg}$ wet weight. Microspheres were $5 \mathrm{mg}$ to $10 \mathrm{mg}$ wet weight. Each data set included BSA standards, and all protein concentrations were generated by individual BSA standard curve.

\section{Hydrogel homogenate production/protein binding}

Hydrogels were made by adding $150 \mu \mathrm{L}$ PEGDA (MW 575, Sigma Aldrich) to varying concentrations of glutathione (Sigma Aldrich, Alfa Aesar) and 0.05\% w/v Irgacure 2959 (Ciba). PBS was used to bring total volumes to $1 \mathrm{~mL}$. The tubes were cured overnight under a UV light (purchased from a local hardware store, $0.25 \mathrm{~mW} / \mathrm{cm}^{2} 254 \mathrm{~nm}$, measured on UVX radiometer). Gels were homogenized with a polypropylene EPPI-pestle homogenizer in the eppendorf tube for 10 to $20 \mathrm{~s}$ at room temperature until most of the homogenate pieces were small enough to pass through a $1 \mathrm{~mL}$ micropipette tip. These homogenates were then washed with $10 \mathrm{~mL}$ PBS in scintillation vials (at least 5 buffer changes over two days).

\section{Microsphere production}

Microspheres were produced by a modified reverse emulsion polymerization method [13]. Polymer solution contained $300 \mu \mathrm{L}$ PEGDA $(\mathrm{d}=1.1 \mathrm{~g} / \mathrm{mL}$, Sigma), $600 \mu \mathrm{L}$ PBS, $5 \mu \mathrm{L}$ eosin Y. Polymer solution $(100 \mu \mathrm{L})$ was transferred to glass test tube $(1.5 \mathrm{~mm}$ diameter, $10 \mathrm{~mm}$ length) and $4 \mathrm{~mL}$ of mineral oil was added. The tube was vortexed 10s until the polymer solution formed an emulsion in the oil. Ammonium persulfate (100 $\mu \mathrm{L}, 20 \%$, Sigma) was added while vortexing and continued for $1 \mathrm{~min}$. Tetramethyl ethylene diamine $(100 \mu \mathrm{L}, 100 \%$, TEMED, Acros) was added and vortexing continued for another minute. The tube was 
then left for 5 min until particles settled out, and several milliliters of deionized water were added. After most of the microspheres have settled into the aqueous phase (several minutes), oil was removed from the test tube and the solution containing the microspheres was collected in an eppendorf tube. They were then spun down, removed of their supernatant, and resuspended and washed with several changes of PBS, then washed over night in $5 \mathrm{~mL}$ PBS. Microspheres were compared to Glutathione Agarose purchased from Gold Biotechnology.

\section{Microscopy}

Brightfield and fluorescence microscopy were carried out on an Olympus IX70 inverted microscope. Micrographs were captured on a Q-imaging Retiga 1300 CCD camera.

\section{Statistical analysis}

Statistical analysis was done using one tailed students ttest for pair wise comparison or ANOVA followed by post-hoc Tukey test for multiple sample comparisons. Significance was set at $\alpha$ less than or equal to 0.05 . Each experiment was independently repeated three times and data is presented as mean plus or minus standard deviation unless otherwise noted.

\section{Abbreviations \\ GST, Glutathione s-transferase; GSH, Reduced glutathione; GFP, Green fluorescent protein; BF, Brightfield; PEGDA, Poly(ethylene glycol) diacrylate.}

\section{Competing interests}

The authors declare that they have no competing interests.

\section{Authors' contributions}

RAG and JSB managed and coordinated the project. JSB, JER, MK, and RAG contributed to the design of the experiments and analysis of the data. All authors participated in the writing and editing of the manuscript. All authors read and approved the final manuscript.

\section{Acknowledgements}

This investigation was funded in part by the National Institutes of Health through grants R01 NS055095 (RAG) and in a facility constructed with support from Research Facilities Improvement Program Grant C06 RR15482 from the National Center for Research Resources, NIH. We would like to thank Dr. Michael E Johnson for providing us with pGex $6 p-1$, the laboratory of Dr. William T. Beck for the use of several reagents, and Dr. Seungpyo Hong and Ryan Pearson for helpful discussions of experiments for this manuscript.

\section{Author details}

'Department of Biopharmaceutical Sciences, University of Illinois, Chicago, IL 60612-7231, USA. ${ }^{2}$ Department of Bioengineering, University of Illinois, Chicago, IL 60607-7052, USA. ${ }^{3}$ Department of Ophthalmology and Visual Science, University of Illinois, Chicago, IL 60612-4319, USA.

Received: 6 June 2012 Accepted: 17 September 2012 Published: 18 September 2012

\section{References}

1. Jackson DA, Symons RH, Berg P: Biochemical method for inserting new genetic information into DNA of simian virus 40: circular SV40 DNA molecules containing lambda phage genes and the galactose operon of Escherichia coli. 1972. Biotechnology 1992, 24:11-16.
2. King TP: Separation of proteins by ammonium sulfate gradient solubilization. Biochemistry 1972, 11(3):367-371.

3. Ghosh R, Cui ZF: Purification of lysozyme using ultrafiltration. Biotechnol Bioeng 2000, 68(2):191-203.

4. Cuatrecasas P: Protein purification by affinity chromatography. Derivatizations of agarose and polyacrylamide beads. J Biol Chem 1970, 245(12):3059-3065

5. Young CL, Britton ZT, Robinson AS: Recombinant protein expression and purification: a comprehensive review of affinity tags and microbial applications. Biotechnol J 2012, 7(5):620-634.

6. Smith DB, Johnson KS: Single-step purification of polypeptides expressed in Escherichia coli as fusions with glutathione S-transferase. Gene 1988, 67(1):31-40.

7. Inoue M, Hara M, Nagashima F, Matsui S, Mitsuyasu N, Morino Y: Affinity chromatography of hepatic glutathione S-transferases on omegaaminoalkyl sepharose derivatives of glutathione. Biochim Biophys Acta 1981, 659(2):362-369.

8. Spana C, Fargnoli J, Bolen JB: In Expression system for Lck protein. Edited by Office EP. EU: BRISTOL-MYERS SQUIBB COMPANY; 2000. EP 0646646 B1.

9. Huang XB, Cyrille D, Thomas P, Bulmus V: Synthesis of Hetertelechelic polymers with affinity to glutathione-s-transferase and biotin-tagged proteins by RAFT polymerization and thiol-ene reactions. Polymer Chemistry 2011, 2:1505-1512.

10. Heim R, Cubitt AB, Tsien RY: Improved green fluorescence. Nature 1995, 373(6516):663-664

11. Pompella A, Visvikis A, Paolicchi A, De Tata V, Casini AF: The changing faces of glutathione, a cellular protagonist. Biochem Pharmacol 2003, 66(8):1499-1503.

12. Dirr H, Reinemer P, Huber R: X-ray crystal structures of cytosolic glutathione S-transferases. Implications for protein architecture, substrate recognition and catalytic function. Eur I Biochem 1994, 220(3):645-661.

13. Franco $\mathrm{CL}$, Price J, West $\mathrm{J}$ : Development and optimization of a dual-photoinitiator, emulsion-based technique for rapid generation of cell-laden hydrogel microspheres. Acta Biomater 2011, 7(9):3267-3276.

14. Tauro JR, Lee BS, Lateef SS, Gemeinhart RA: Matrix metalloprotease selective peptide substrates cleavage within hydrogel matrices for cancer chemotherapy activation. Peptides 2008, 29(11):1965-1973.

15. Tauro JR, Gemeinhart RA: Extracellular protease activation of chemotherapeutics from hydrogel matrices: A new paradigm for local chemotherapy. Mol Pharm 2005, 2(5):435-438.

16. Tauro JR, Gemeinhart RA: Matrix metalloprotease triggered local delivery of cancer chemotherapeutics. Bioconjugate Chem 2005, 16(5):1133-1139.

17. Vartak DG, Gemeinhart RA: In vitro evaluation of functional interaction of integrin av $\beta 3$ and matrix metalloprotease-2. Mol Pharm 2009, 6(6):1856-1867.

18. Ross $A E$, Tang MY, Gemeinhart RA: Effects of molecular weight and loading on matrix metalloproteinase- 2 mediated release from poly(ethylene glycol) diacrylate hydrogels. AAPS J 2012, 14(3):482-490

19. Zhang Y, Gemeinhart RA: Improving matrix metalloproteinase-2 specific response of a hydrogel system using electrophoresis. Int J Pharm 2012, 429(1-2):31-37.

20. Kopecek J: Smart and genetically engineered biomaterials and drug delivery systems. Eur J Pharm Sci 2003, 20(1):1-16.

21. Raps SP, Lai JC, Hertz L, Cooper AJ: Glutathione is present in high concentrations in cultured astrocytes but not in cultured neurons. Brain Res 1989, 493(2):398-401.

22. Vartak D, Gemeinhart RA: Matrix metalloproteases: underutilized targets for drug delivery. J Drug Target 2007, 15(1):1-21.

23. Overall CM, Kleifeld O: Validating matrix metalloproteinases as drug targets and anti-targets for cancer therapy. Nat Rev Cancer 2006 6(3):227-239.

24. Turk B: Targeting proteases: successes, failures and future prospects. Nat Rev Drug Discov 2006, 5(9):785-799.

25. Turk BE, Huang LL, Piro ET, Cantley LC: Determination of protease cleavage site motifs using mixture- based oriented peptide libraries. Nat Biotechnol 2001, 19(7):661-667. 
26. Kratz F, Drevs J, Bing G, Stockmar C, Scheuermann K, Lazar P, Unger C: Development and in vitro efficacy of novel MMP2 and MMP9 specific doxorubicin albumin conjugates. Bioorg, Med Chem Let 2001,

11(15):2001-2006.

27. Bradford MM: A rapid and sensitive method for the quantitation of microgram quantities of protein utilizing the principle of protein-dye binding. Anal Biochem 1976, 72:248-254.

doi:10.1186/1472-6750-12-63

Cite this article as: Buhrman et al.: In-house preparation of hydrogels for batch affinity purification of glutathione $S$-transferase tagged recombinant proteins. BMC Biotechnology 2012 12:63.

\section{Submit your next manuscript to BioMed Central and take full advantage of:}

- Convenient online submission

- Thorough peer review

- No space constraints or color figure charges

- Immediate publication on acceptance

- Inclusion in PubMed, CAS, Scopus and Google Scholar

- Research which is freely available for redistribution 